\title{
Ações e estratégias voltadas para a ciência aberta em universidades estaduais paulistas: um estudo multicaso
}

\author{
Nivaldo Calixto Ribeiro \\ Doutorando; Universidade Federal de Minas Gerais, Belo Horizonte, MG, Brasil. \\ nivaldo@ufla.br \\ Dalgiza Andrade Oliveira \\ Doutora; Universidade Federal de Minas Gerais, Belo Horizonte, MG, Brasil \\ dalgizamg@gmail.com \\ Sarah Rúbia de Oliveira Santos \\ Mestranda; Universidade Federal de Minas Gerais, Belo Horizonte, MG, Brasil. \\ sarahrubia@ufmg.br
}

Resumo: Este estudo tem como objetivo geral identificar e refletir sobre as ações das universidades estaduais paulistas - Universidade de São Paulo (USP), Universidade Estadual Paulista "Júlio de Mesquita Filho" (Unesp) e Universidade Estadual de Campinas (Unicamp) - quanto à divulgação da pesquisa científica e à produção de novos conhecimentos por meio de estratégias voltadas para a Ciência Aberta. Como objetivos específicos, pretende-se apontar políticas públicas adotadas nas universidades estaduais paulistas e identificar como essas instituições definem suas diretrizes relacionadas às novas formas de compartilhamento de dados e de informação. Como procedimentos de coletas de dados, são empregados a pesquisa bibliográfica e o levantamento documental. Além disso, endereçamos aos gestores das três universidades aqui trabalhadas, via Serviço de Informações ao Cidadão (SIC), uma solicitação de compartilhamento de informações sobre as iniciativas e as estratégias de Ciência Aberta desenvolvidas por essas instituições. Para a análise, categorizamos e classificamos os dados levantados por meio das cinco correntes de pensamento que representam perspectivas reconhecidas pelos autores Fecher e Friesike (2013). Os dados foram tratados em planilha Excel e interpretados com o auxílio do conjunto de ferramentas nas nuvens de business intelligence Power BI. Concluiu-se que as universidades investigadas apresentam grande foco na pesquisa e, portanto, estão seriamente envolvidas em diversos movimentos voltados para a Ciência Aberta.

Palavras-chave: Ciência Aberta. Universidades Estaduais Paulistas - Brasil. Comunicação Científica. 


\section{Introdução}

A forma como se faz ciência não deve parar de evoluir ou ser interrompida. Atualmente, vive-se o quarto paradigma da pesquisa, caracterizado pelo volume de dados oriundos das mais diversas fontes, captados por meio de aparatos, de recursos tecnológicos e da Internet (OLIVEIRA; SILVA, 2016). Segundo Silva e Silveira (2019), a massificação e a capacidade de expandir o acesso a dados e informações resultantes de pesquisas acadêmicas, por meio da Web, impulsionaram o movimento de livre acesso à informação, originando um novo ecossistema e incorporando vários movimentos em um só, este conhecido como Ciência Aberta. Muitos desses movimentos tiveram marco em projetos iniciados e desenvolvidos, por meio de decisões administrativas, em universidades, sem o envolvimento direto em políticas ou diretrizes governamentais.

A Ciência Aberta é um termo guarda-chuva que permite múltiplas interpretações, pois engloba diversas práticas distintas e abordagens (ALBAGLI, 2014). A perspectiva abordada neste estudo é a de Albagli (2014) ao descrever que a Ciência Aberta pode promover ampliação do conhecimento público, possibilitando o aumento dos índices de produtividade científica e de inovação, além do crescimento das taxas de retornos sociais dos investimentos em ciência e tecnologia. É importante considerar o que Deng (2008) destaca ao afirmar que a maior parte das pesquisas científicas é desenvolvida em universidades e instituições públicas de pesquisa, financiadas pelo Estado e/ou agências governamentais de fomento e que, portanto, seus resultados não devem ser privados.

O estudo de Oliveira e Silva (2016) aponta que, no período de sua produção, a realidade brasileira voltada para a Ciência Aberta era considerada incipiente. Observa-se um questionamento sobre o papel da ciência e sua efetividade, quanto ao retorno de seus resultados de pesquisa para a sociedade de forma organizada, acessível e segura, especialmente quando se trata de financiamento com recursos públicos. Marques (2016) explica que essa cobrança pode ser considerada comum, especialmente em tempos de crise econômica, quando os anseios se voltam para a aplicação do recurso público em atividades 
que deem retorno visível e imediato. Embora algumas iniciativas sejam percebidas, não se pode afirmar quais universidades estão engajadas com essa demanda da sociedade, incentivada por meio da cobrança das agências de fomento, que passaram a exigir comprovação das pesquisas financiadas. Ao analisar esse cenário, percebe-se que as atuais iniciativas precisam ser conduzidas por uma política ou por diretrizes que apoiem as práticas da Ciência Aberta (OLIVEIRA; SILVA, 2016), o que presume uma produção científica colaborativa e compartilhada de forma mais eficiente.

Diante do exposto, este estudo questiona: quais as políticas e as iniciativas das universidades estaduais paulistas - Universidade de São Paulo (USP), Universidade Estadual Paulista "Júlio de Mesquita Filho" (Unesp) e Universidade Estadual de Campinas (Unicamp) - centradas no conhecimento e no acesso aberto, voltadas para tornar a ciência acessível ao cidadão? Quais ações e ferramentas são adotadas para disponibilizar o conhecimento de forma gratuita ao público? Que mecanismos essas universidades estão utilizando para tornar a criação do conhecimento mais eficiente e mais orientada? Como são avaliadas as contribuições científicas? Quais os sistemas métricos utilizados por essas instituições para mensurar o impacto científico de suas pesquisas?

Assim, este estudo tem como objetivo geral identificar e refletir sobre as ações das universidades estaduais paulistas - USP, Unesp e Unicamp - quanto à divulgação da pesquisa científica e à produção de novos conhecimentos por meio de estratégias voltadas para a Ciência Aberta. Como objetivos específicos, pretende-se apontar políticas públicas adotadas nas universidades estaduais paulistas e identificar como essas instituições estão definindo suas diretrizes relacionadas às novas formas de compartilhamento de dados e da informação.

As universidades que integram o corpus desta investigação foram selecionadas em função de sua representatividade para o ensino, a pesquisa e a extensão. As três instituições aparecem com frequência em diversos rankings nacionais e internacionais ${ }^{1}$. Desta maneira, entende-se que essas universidades exercem um papel preponderante para a comunicação e a divulgação da pesquisa científica, o que vai ao encontro de ações voltadas para a disseminação do conhecimento por meio da Ciência Aberta. 


\section{Escolas de pensamento da ciência aberta}

A Ciência Aberta pode ser considerada como processo meio e não o fim (OLIVEIRA; SILVA, 2016). Em um estudo baseado na revisão de literatura, Fecher e Friesike $(2013 ; 2014)$ propuseram cinco escolas de pensamento que representam perspectivas diferentes e complementares. A seguir, essas cinco escolas de pensamentos serão descritas, no intuito de se aprofundar as questões levantadas pelos autores e de embasar a discussão dos resultados deste trabalho.

\subsection{Escola Pública (Public School)}

A Escola Pública (Public School) prevê pesquisas científicas que incluam em sua prática não só os especialistas, mas também o cidadão comum, pois defende-se que a ciência deve estar acessível ao público em geral (FECHER; FRIESIKE, 2013; ALBAGLI; CLÍNIO; RAYCHTOCK, 2014). Segundo Fecher e Friesike (2013), os pressupostos básicos, as tecnologias da Web social e da Web 2.0 estão situados em duas vertentes: a primeira é a acessibilidade para o processo de pesquisa/abertura e a segunda é a compreensibilidade dos resultados da pesquisa, ou melhor, a preparação do produto da pesquisa para um público de não especialistas. Ambas as perspectivas envolvem a relação entre os cientistas e o público para definir a abertura como uma forma de aproximação entre a comunidade científica e a sociedade.

Uma das ferramentas e métodos adotados por esta Escola é a Ciência Cidadã, uma forma de ciência baseada na participação de cidadãos que geram e analisam grandes quantidades de dados, partilham o seu conhecimento, discutem e apresentam os resultados de projetos (RUIZ-MALLÉN et al., 2016). Os autores entendem que a Ciência Cidadã pode aumentar a compreensão das pessoas sobre a ciência e, ao mesmo tempo, ajudar os cientistas a conduzirem suas pesquisas. Para Hecker et al. (2018), o aumento de projetos envolvendo a Ciência Cidadã demonstra o desejo do público em geral de participar mais ativamente na produção de conhecimento. Concomitantemente, cientistas, pesquisadores, organizações e financiadores de pesquisa estão descobrindo os benefícios de abri- 
la para a sociedade. Este parece não ser apenas um papel passivo, mas ativamente definido na agenda, nos crowdsourcings ${ }^{2}$ via plataformas Web, coletando e analisando dados científicos.

Os festivais ou eventos destinados a promover a aprendizagem da ciência informal e o engajamento do público em várias formas de interação que compõem a comunicação da ciência institucional podem ser considerados como Science Public Relations (PR), Ciência PR ou simplesmente relações públicas em ciências. Nesse caso, segundo as orientações do Guidelines for Good Science PR, a Ciência PR parece ser entendida como o modo pelo qual as instituições moldam conscientemente a comunicação com os parceiros de diálogos internos e externos, buscando a melhor compreensão de suas ações (SIGGENER KREIS, 2013).

Outra ferramenta citada como um método desejável para a prática da Ciência Aberta, com características da Escola Pública, é a Science blogging. Os blogs de ciência existem desde o início dos anos 2000 (ZIVKOVIC, 2012) e, nos últimos anos, a plataforma de microblogging Twitter e outros canais de mídia social, que exigem menos tempo de manutenção do que um blog completo, ameaçavam torná-los obsoletos. Entretanto, alguns cientistas mantêm os blogs e estes continuam a desempenhar um papel importante estimulando as colaborações, transmitindo informações cruciais e fortalecendo as comunidades científicas (BROWN; WOOLSTON, 2018).

Embora apenas algumas das ferramentas, ações e métodos que podem ser enquadrados por essa escola sejam citados neste texto, em determinadas situações poderão ser adotados outros modelos, iniciativas ou pesquisas em diferentes perspectivas e contextos, favorecendo o movimento em prol da Ciência Aberta em diversos segmentos.

\subsection{Escola Democrática (Democratic School)}

A Escola Democrática (Democratic School) está preocupada com o acesso ao conhecimento, pois o considera um direito humano, condição que se torna ainda mais desejável quando a pesquisa científica conta com financiamento público (FECHER; FRIESIKE, 2013; ALBAGLI; CLÍNIO; RAYCHTOCK, 2014). De acordo com Fecher e Friesike (2013), a razão pela qual existe o discurso sobre o 
livre acesso a produtos de pesquisa emerge da lógica de que todos deveriam ter o mesmo direito ao conhecimento.

Nas palavras de Albagli, Clínio e Raychtock (2014) essa escola se apoia em duas estratégias: uma voltada para a via de dados abertos, que busca a disponibilização dos dados primários, coletados durante a pesquisa de maneira que possibilitem não apenas a sua consulta, mas efetuar análise e reutilização em pesquisas futuras. A outra, segundo Fecher e Friesike (2013) e Albagli, Clínio e Raychtock (2014), voltada para o acesso aberto aos resultados de pesquisa, tornados públicos por meio de artigos publicados em revistas e anais de eventos científicos, além de representações digitais de materiais, pinturas, imagens, gráficos ou material multimídia.

Conforme Santos (2013, p. 2), “[...] a democratização e a universalização do acesso ao conhecimento nas ciências e humanidades é condição fundamental para o desenvolvimento igualitário e sustentável das nações.". Nas palavras do autor, “[...] o seu estabelecimento objetiva garantir à sociedade o acesso gratuito, público e aberto ao conteúdo integral da produção intelectual desenvolvida pelas universidades ou outras instituições de pesquisa.” (SANTOS, 2013, p. 2).

Para uma sociedade democrática com características que enfatizam a globalização, o compartilhamento, a colaboração e o ambiente digital, os direitos de propriedade intelectual vigentes da publicação científica precisam ser revistos e mais bem compreendidos, buscando um novo fazer científico no cenário da ciência contemporânea, a qual conduz à investigação científica aberta e pública (OLIVEIRA; GUIMARÃES; KOSHIYAMA, 2019). Essa é uma das pautas constantes da Escola Democrática.

\subsection{Escola Pragmática (Pragmatic School)}

A Escola Pragmática (Pragmatic School) trabalha com uma noção de aberto mais próxima da inovação aberta e vislumbra que o processo científico pode ser otimizado pela incorporação do conhecimento externo e a colaboração por meio de ferramentas on-line (FECHER; FRIESIKE, 2013; ALBAGLI; CLÍNIO; RAYCHTOCK, 2014). Acompanha o princípio de que a produção do conhecimento é mais eficiente se desenvolvida em colaboração e fortalecida por 
meio da crítica. Essa escola procura aproveitar os efeitos da rede de contato para estabelecer a ligação entre pesquisadores, tornando os métodos investigativos transparentes e eficientes (FRANCK et al., 2018).

Surowiecki (2004) foi um dos precursores a tratar da inovação aberta. Em seu texto A Sabedoria das Multidões, o autor exemplifica como esse fenômeno ocorre. O autor relata a história do primo de Charles Darwin, Francis Galton, autoridade detentora de muitos conhecimentos, e que, em uma feira de gado realizada em 1906, foi pioneiro em atentar para a sabedoria das multidões. Os visitantes dessa feira tiveram a oportunidade de adivinhar o peso de um boi e a pessoa com o palpite mais próximo do peso real ganharia um prêmio. Galton registrou e fez a média de todas as inscrições do país no concurso "Adivinhe o peso do boi" e foi incrivelmente preciso, superando não apenas a maioria das proposições individuais, mas também as de especialistas em gado. Essa é a essência da sabedoria das multidões - seu julgamento comum converge para a solução certa (KADIKAR, 2019).

Em diversos contextos, Laé (2016) explica que investidores, designers, profissionais de marketing e o público em geral podem ser convidados a colaborar, trazendo ideias para determinado projeto com o objetivo de se ter perspectivas externas para auxiliar na criação e no desenvolvimento de um negócio ou contribuir com qualquer outra solução.

\subsection{Escola da Infraestrutura (Infrastructure School)}

A Escola da Infraestrutura (Infrastructure School) está preocupada com as possibilidades e os desafios tecnológicos que permitem práticas emergentes de pesquisa na Internet, e que são necessários às práticas da Ciência Aberta, especialmente quando se trata de ferramentas, aplicativos de software e redes de computação (FECHER; FRIESIKE, 2013). Para os autores, a literatura sobre esse assunto geralmente é específica para cada caso e se concentra nos requisitos tecnológicos para projetos específicos.

A Escola da Infraestrutura observa tendências como a computação distribuída por meio da conexão de diversos computadores para formar uma rede de alto desempenho no processamento de pesquisas com uso intensivo de dados. 
Como exemplo, o projeto Folding@home, que prevê a utilização de parte do processamento de dados de computadores de voluntários para realizar cálculos e simulações destinadas à pesquisas médicas, entre elas: Esclerose lateral amiotrófica, Influenza, Câncer e COVID. Outra face da dimensão da infraestrutura está relacionada à constituição de redes sociais de colaboração para promover mais interação e colaboração entre cientistas e na disponibilização de recursos da web2.0, com a finalidade de criação de um ambiente social virtual de pesquisa (FECHER; FRIESIKE, 2013; CARUSO, 2015; OLIVEIRA; SILVA, 2016).

\subsection{Escola das Métricas (Measurement School)}

A Escola das Métricas (Measurement School) busca novas formas de mensurar a produção científica, uma vez que esta tende a migrar para ambientes on-line e adotar novos formatos de publicação (FECHER; FRIESIKE, 2013). Segundo os autores, essa escola está preocupada com padrões alternativos para determinar o impacto científico, pois é indiscutível que o número médio de citações de um artigo de uma revista pode influenciar decisivamente na reputação de um pesquisador, no financiamento e nas oportunidades da sua carreira.

Para Souza (2015), por meio das métricas alternativas, há a possibilidade de se obter informações a respeito do alcance e do uso de trabalhos científicos, algo complexo de se conseguir com processos tradicionais, tais como a análise de citação. A citação é um indicador muito relevante e de credibilidade adotado pela academia, porém, existem outras abordagens com grande potencial para verificar o impacto das pesquisas científicas.

Existem diversas métricas como a bibliometria, a webometria, a altmetria, a semantometria, que vêm ganhando popularidade no meio acadêmico. Embora as métricas citadas tenham como objetivo demonstrar sobremaneira o impacto das publicações científicas, cada uma delas tem suas peculiaridades. A bibliometria envolve estudos que procuram quantificar os processos de comunicação escrita, aplicando técnicas numéricas específicas (FORESTI, 1991), como métodos estatísticos e matemáticos na análise de obras literárias (PRITCHARD, 1969). A webometria é o estudo de aspectos quantitativos de construção e uso de recursos 
informacionais, estruturas e tecnologias da Web a partir de abordagens bibliométricas e informétricas (BJÖRNEBORN, 2004). Mais recentemente, surgiu a altmetria com foco na análise da comunicação científica no contexto da Web social e dos recursos da Web 2.0 (PRIEM et al, 2010; GOUVEIA, 2013). A altmetria pode atuar como métrica complementar e auxiliar na construção de quadros mais completos sobre o impacto da ciência. Já a semantometria, considerada como uma extensão da bibliometria, da webometria e da altmetria, é uma categoria de métrica de avaliação de pesquisa que se baseia na premissa de que é necessário o texto completo para avaliar o valor de uma publicação, utilizando-se de representação semântica (KNOTH; HERRMANNOVA, 2014).

\section{Opções metodológicas}

Nesta seção, elencam-se os procedimentos metodológicos para a realização do estudo, bem como as estratégias utilizadas para responder às questões investigativas e atender aos objetivos geral e específicos, apresentando uma breve descrição da coleta de dados utilizada.

\subsection{Caracterização do estudo}

Esta investigação trata-se de uma abordagem qualitativa, a qual inclui a reflexão dos pesquisadores, uma descrição e interpretação do problema e a sua contribuição para a literatura ou um chamado à mudança (CRESWELL, 2014). Para esse autor, a pesquisa qualitativa começa com pressupostos e uso de estruturas interpretativas/teóricas que informam o estudo dos problemas da pesquisa, abordando os significados que os indivíduos ou grupos atribuem a um problema social ou humano.

O estudo pode ser classificado como descritivo e exploratório, pois foi aplicado um questionário semiestruturado. Ainda recorreu à literatura e a fontes documentais, como sites institucionais e publicações das próprias universidades, no intuito de levantar dados qualitativos para verificar o uso de práticas de Ciência Aberta e sua contextualização nas universidades estaduais paulistas.

\subsection{Procedimento de coleta de dados e tratamento dos dados}


Para a coleta de dados, foi encaminhado um questionário semiestruturado, endereçado aos representantes máximos, reitores ou representantes interinos de três universidade estaduais paulistas, via Serviço de Informações ao Cidadão (SIC), conforme exposto no Quadro 1.

Quadro 1 - Síntese das informações requisitada às universidades para o levantamento de práticas de Ciência Aberta

\section{ABORDAGEM DAS REQUISIÇÕES SIC}

- existência de iniciativas de Ciência Cidadã promovidas pela universidade;

- disponibilidade de repositórios institucionais e link de acesso;

- existência de Política Institucional de Informação e link de acesso ao documento;

- disponibilidade de um Portal de Periódicos Científicos Institucional e do link de acesso;

- lista de periódicos científicos vinculados a universidade, disponíveis em acesso aberto e a identificação da plataforma de gestão das publicações;

- forma de avaliação científica adotada pelos periódicos vinculados à universidade;

- existência de política de produção de artigos compartilhada;

- forma de atuação da universidade com recursos educacionais abertos;

- disponibilidade de política de dados abertos e do link de acesso ao documento;

- existência de incentivos ao pesquisador para publicar em acesso aberto;

- existência de projetos de inovação aberta, com uso de plataformas de crowdsourcing ou outros meios para solucionar problemas ou criar novos produtos;

- formas de controlar ou acompanhar as métricas formais da produção científica dos pesquisadores da instituição e como é realizado este processo.

- existência de monitoramento do uso de métricas alternativas;

- forma da universidade divulgar as suas publicações científicas por meio de mídias sociais - existência de algum órgão responsável pela divulgação científica.

Fonte: Dados da pesquisa (2020)

Os resultados foram utilizados para confrontar a teoria e a prática relacionadas à Ciência Aberta. As questões foram elaboradas tendo como referências os itens de representação e as perspectivas das escolas ou correntes de pensamento da Ciência Aberta propostas por Fecher e Friesike $(2013 ; 2014)$ a saber: Pública, Democrática, Pragmática, Infraestrutura, Métricas e os desdobramentos da Ciência Aberta. As requisições SIC foram relevantes para identificar as ações desenvolvidas pelas universidades estaduais paulistas.

Segundo a Lei n. ${ }^{\circ}$ 12.527, de 18 de novembro de 2011, conhecida como Lei de Acesso à Informação (LAI), SIC é uma unidade física que deve existir em todos os órgãos e entidades do poder público, em local identificado e de fácil acesso, para atender o cidadão que deseja solicitar o acesso à informação pública (BRASIL, 2011). De acordo com a Controladoria Geral da União, os SICs têm como objetivos: atender e orientar o público quanto ao acesso a informações; 
conceder o acesso imediato à informação disponível; informar sobre a tramitação de documentos nas suas respectivas unidades e protocolizar documentos e requerimentos de acesso a informações (BRASIL, 2017).

A Lei Federal n. ${ }^{\circ}$ 12.527, de 18 de novembro de 2011, e o Decreto do Estado de São Paulo n. ${ }^{0}$ 58.052, de 16 de maio de 2012, regulamentam o direito, previsto na Constituição, de qualquer pessoa solicitar e receber dos órgãos e entidades públicos, de todos os entes e Poderes, informações públicas por eles produzidas ou custodiadas. Considerando os objetivos propostos nesta pesquisa, esse canal formal e legal de comunicação traduz-se em uma excelente ferramenta e estratégia de coleta de dados, visto ainda que, conforme a lei prevê, se a informação estiver disponível, ela deve ser entregue imediatamente ao solicitante. Caso não seja possível conceder o acesso imediato, o órgão ou entidade tem até 20 dias para atender ao pedido, prazo que pode ser prorrogado por mais 10 dias, se houver justificativa expressa. No universo da pesquisa, o prazo previsto para gerir a coleta de informação é bastante razoável, além de as respostas estarem formalmente protocoladas e em condições de serem publicizadas em repositórios de dados abertos para posterior acesso público, se for o caso.

Para a compreensão da coleta de dados, entende-se que é importante historiar o processo junto às universidades. No dia 21 de outubro de 2019, os três formulários de requisição de informações relacionadas às práticas da Ciência Aberta foram enviados às universidades, por meio dos sistemas SIC-Unesp ${ }^{3}$, SICUSP $^{4}$ e SIC-Unicamp ${ }^{5}$. Com o prazo inicial de 20 dias para o atendimento, a previsão de retorno era para 10 de novembro de 2019. Foram gerados protocolos para acompanhamento das solicitações.

No dia 23 de outubro, o SIC-Unesp recusou o pedido de informação, alegando que tal manifestação não se tratava de uma solicitação de informação, mas sim de uma colaboração em pesquisa acadêmica. Conforme a Portaria Unesp n. ${ }^{\circ} 257$, de 25 de julho de 2019, que regulamenta, no âmbito daquela universidade, a Lei n. $^{\circ} 12.527$, de 18 de novembro de 2011, que regula o acesso a informações, em seu art. $5^{\circ} . \S 1^{\circ}$, IV, o SIC-Unesp “[...] deverá realizar o serviço de busca e fornecimento de documentos, dados e informações sob custódia da Unesp ou fornecer ao requerente orientação sobre o local onde encontrá-los.”. Embora o 
protocolo tenha sido recusado, foi orientado que se encaminhasse a solicitação diretamente ao e-mail da Coordenadoria Geral de Bibliotecas. No mesmo dia, um e-mail com a solicitação de informação foi enviado para o setor indicado, que prontamente respondeu, enviando as informações no dia 31 de outubro de 2019.

No dia 21 de novembro, o SIC-USP retornou informando que aquele canal é destinado ao acesso a documentos e informações pré-existentes, ou de produção imediata, relacionados à administração do patrimônio público, utilização de recursos públicos, licitações, contratos administrativos etc. e, assim como o SICUnesp, orientou a entrar em contato com Sistema Integrado de Bibliotecas USP. No dia 28 de novembro de 2019, a Agência USP de Gestão da Informação Acadêmica (Aguia), conhecida como Sistema Integrado de Bibliotecas da USP, enviou as informações solicitadas.

Cabe mencionar que, apesar de a requisição das informações sobre a Ciência Aberta enviada às universidades ter o objetivo de coletar dados e informações para uma pesquisa acadêmica, entende-se que se trata de pedido de informações de interesse público, devido à relação com pesquisas científicas desenvolvidas pela instituição com recursos públicos no todo ou em parte. Ressalta-se ainda que as informações solicitadas foram endereçadas às reitorias, pois não envolviam, exclusivamente, dados ou atividades relacionadas à biblioteca, mas sim a políticas institucionais de divulgação, comunicação e abertura da ciência, o que abarca a instituição como um todo.

Com relação ao Protocolo SIC-Unicamp, no dia 11 de novembro de 2019, foi solicitada a prorrogação do prazo de entrega, justificado pelo volume de informações requeridas e a necessidade de coleta de dados em diferentes órgãos. No dia 2 de dezembro de 2019, o atendimento foi concluído e as informações foram enviadas por e-mail. Note-se que, diferentemente das outras universidades, o protocolo SIC-Unicamp foi respondido por quatro órgãos administrativos: Sistema de Bibliotecas (SBU/Unicamp), Pró-Reitoria de Pesquisa (PRP/Unicamp), Grupo Gestor de Tecnologias Educacionais (GGTE/Unicamp) e Agência de Inovação (Inova/Unicamp). Assim, os autores deste estudo entenderam que as respostas enviadas pela Coordenadoria Geral de Bibliotecas da Unesp e pela Aguia/USP compõem as respostas dos SICs de suas instituições 
e as menções relacionadas às suas instituições serão representadas por SIC-Unesp e SIC-USP.

Após a coleta, os dados foram importados para o Power BI, no formato de planilha, para as análises posteriormente realizadas. Conforme Passos (2016), o Power BI pode ser considerado como um conjunto de ferramentas de Business Intelligence na nuvem para análise de dados e compartilhamento de ideias, possibilitando o monitoramento desses dados de diferentes fontes por meio de dashboard. Nas palavras de Gomes (2017, documento eletrônico), “[...] dashboard é um painel visual que apresenta, de maneira centralizada, um conjunto de informações: indicadores e suas métricas.".

\section{Resultados e discussão}

No intuito de facilitar o entendimento e a clareza dos dados, foi efetuada uma análise de conteúdo, conforme proposto por Oliveira (2008), que prevê três etapas: 1. Pré-análise; 2. Exploração do material ou codificação e; 3. Tratamento dos resultados, inferência. Somadas às interpretações das cinco correntes de pensamento estabelecidas por Fecher e Friesike (2013; 2014), alinhadas aos desdobramentos da Ciência Aberta.

Iniciando com a Escola Pública, foram abordados projetos e iniciativas de pesquisa envolvendo a Ciência Cidadã, comumente confundida com a extensão universitária. Embora ambas envolvam a participação da sociedade, há distinção entre elas. A extensão universitária é composta de ações das universidades junto às comunidades na quais estão inseridas e, assim, são convocadas ao aprofundamento de seu papel como agentes de transformação social. Isso aproxima a produção e a transmissão de conhecimento adquirido com o ensino e a pesquisa de seus efetivos destinatários, podendo corrigir, nesse processo, os entraves que tornam assimétrica e desigual a apropriação social do conhecimento, das ciências e também das tecnologias (PAULA, 2013). Com relação à Ciência Cidadã, essa pode ser considerada como o engajamento de membros da sociedade em atividades de pesquisa de forma voluntária.

Na USP, além das atividades de extensão na Estação Ciência, Museus e Hospitais Universitários, a instituição dispõe da participação de vários integrantes 
da sociedade nas pesquisas. Na Unicamp, pesquisas aplicando os conceitos de Ciência Cidadã acontecem a partir da iniciativa dos próprios pesquisadores envolvidos. Na Unesp, há algumas iniciativas, como o Projeto Pedagógico Cidadão, o PEC - Formação Continuada e outras. Um bom exemplo de pesquisa no contexto da Ciência Cidadã é o da pesquisadora Karlla V. C. Barbosa ${ }^{6}$, da Unesp em Rio Claro. Segundo Jorge (2019, documento eletrônico), do Portal de Notícias da Unesp, “[...] nesta pesquisa, aves populares de fácil identificação foram equipadas com 'mochilas de GPS' para entender o seu processo de migração e qual o impacto que a urbanização exerce sobre ele.”. O monitoramento das aves foi realizado com a colaboração de cidadãos que entraram em contato com a pesquisadora notificando ter encontrado aves anilhadas em áreas verdes das cidades de São Paulo, Guarulhos ou Rio Claro (JORGE, 2019).

Outra perspectiva da escola de pensamento pública, a Ciência PR, pode ser observada nas instituições, pois são realizados eventos diversos para aproximar prática científica e sociedade. A Unesp realiza a Feira de Projetos Complementares com a participação de projetos de extensão, grupos de pesquisa e empresas juniores; a USP promove a Feira de Ciência e Tecnologia; a Unicamp realiza Ciência na Praça, atividade que busca apresentar à população de Campinas pesquisas desenvolvidas na instituição.

Apesar de a Science blogging não ter sido mencionada pelos SIC das três universidades, em consulta aos seus sites oficiais e aos dados levantados, constatou-se que elas dispõem de órgãos, setores (assessoria, superintendência ou agência de comunicação e informação) e canais (sites, perfis em mídias sociais digitais, podcasts) responsáveis pela divulgação da ciência e equipe responsável por traduzir, em determinados casos, pesquisas científicas para uma linguagem mais coloquial, possibilitando a compreensão do conteúdo das pesquisas por qualquer membro da sociedade, e não apenas pelos especialistas.

Com relação à Escola Democrática, a qual tem como ferramentas e métodos acesso aberto, dados abertos, códigos abertos entre outros, tem-se uma consolidada representatividade de ações para esses movimentos nas três universidades. De acordo com Costa e Leite (2017), a expressão "acesso aberto" foi usada pela primeira vez em 2001, quando o Open Society Institute se reuniu e 
estabeleceu o que é conhecido como a Iniciativa de Acesso Aberto de Budapeste, tradução de Budapest Open Access Initiative (BOAI), cujo objetivo era criar um conjunto de recomendações, projetadas para fornecer ao público acesso livre e irrestrito à pesquisa acadêmica. Desde então, a expressão "acesso aberto" foi definida por diferentes grupos de formas distintas.

De acordo com informações extraídas do site da Universidade do Minho (2020), open access, na tradução literal "acesso livre" ou "acesso aberto", significa a disponibilização livre na Internet de cópias gratuitas, on-line, de artigos de revistas científicas revisadas por pares, peer-reviewed, comunicações em conferências, bem como relatórios técnicos, teses e documentos de trabalhos acadêmicos ou de pesquisa. Concordando, Haddaway (2018) expõe que acesso aberto se refere à disponibilização gratuita de literatura de pesquisa na Internet, acessível ao público, podendo ser lida, baixada, copiada, distribuída, impressa, pesquisada, rastreada para indexação e análise de texto sem repercussões ou redução das barreiras legais ou financeiras.

No entendimento de Caruso (2015), pesquisas e publicações em Ciência da Informação têm sido realizadas sobre tendências tecnológicas emergentes, a exemplo do acesso aberto por meio de repositórios e periódicos como instrumentos para publicação e divulgação acadêmica.

As três universidades - Unesp ${ }^{7}$, USP $^{8}$ e Unicamp ${ }^{9}$ - dispõem de Repositório Institucional. Com relação à existência de uma Política Institucional de Informação, a Unesp informou que uma Comissão de Acesso Aberto foi instituída para desenvolver essa política. Na USP, a Resolução n. ${ }^{\circ} 6.444$, de 22 de outubro de 2012, instituiu diretrizes e procedimentos para promover e assegurar a coleta, o tratamento e a preservação da produção intelectual gerada nas unidades da universidade e pelos programas conjuntos de pós-graduação, bem como a disseminação e acessibilidade aos comunitários.

O Repositório Institucional ou a Biblioteca Digital da Produção Intelectual passou a ser o instrumento oficial incumbido de reunir a produção intelectual daquela instituição. Na Unicamp, o repositório da sua produção científica e intelectual teve sua criação oficializada em dezembro de 2015, atendendo a uma demanda da Fundação de Amparo à Pesquisa do Estado de São Paulo (Fapesp), 
em 2013, junto ao conselho de reitores das universidades estaduais paulistas. A Resolução do Gabinete do Reitor n. ${ }^{\circ}$ 013/2015, de 6 de julho de 2015, instituiu a criação do Repositório da Produção Científica e Intelectual da Unicamp. A política para o Repositório Institucional está sendo finalizada. No momento, ele segue algumas diretrizes estabelecidas para nortear os trabalhos, além de ter respaldo na Resolução citada. Fisicamente, o Repositório está sob responsabilidade e gerenciamento da diretoria e tratamento da informação, vinculado ao Sistema de Bibliotecas da Unicamp.

Com relação aos periódicos, na Unesp, todos aqueles vinculados à universidade estão em acesso aberto, com Open Journal System (OJS), e a avaliação é realizada às cegas. Esse formato de avaliação foi selecionado há tempos, pois são títulos já consolidados, todavia, com a expansão da temática da Ciência Aberta, os editores poderão conhecer outras formas de avaliação e poderão optar por elas. Na USP, por exemplo, há 190 títulos de periódicos com acesso aberto e a avaliação científica por pares, na maioria dos títulos, é realizada às cegas. Na Unicamp, a avaliação científica é realizada por pares, na sua totalidade de 30 periódicos, pelo formato duplo-cego, por opção dos editores que gerenciam cada título. Em conformidade com Nassi-Calò (2015, documento eletrônico), a revisão por pares duplo-cego “[...] é quando um periódico disponibiliza aos autores a opção de manter seu nome e afiliação confidenciais durante o processo de revisão dos artigos submetidos.". Ainda na visão da autora, no "[...] processo simples-cego, apenas a identidade dos revisores é mantida em segredo.”. A autora expõe que revisões totalmente abertas (em que a identidade de autores e revisores é conhecida por ambos), revisões abertas publicadas ao final do artigo (possibilitando um espaço para discussões pós-publicação) e a substituição da revisão por pares por revisão pós-publicação, são alternativas para o processo de peer review.

Ao tratar sobre Portal de Periódicos, identificou-se que, na Unesp, cada revista ou conjunto de revistas possui sua própria estrutura de gerenciamento. $\mathrm{Na}$ USP, o Portal de Revistas ${ }^{10}$, criado em 2008, é considerado a biblioteca digital das revistas publicadas por unidades, órgãos de integração, órgãos complementares e, em alguns casos, em parceria oficial com instituições externas. 
O portal foi construído de forma a ser interoperável com sistemas similares nacional e internacionalmente. Na Unicamp, o Portal de Periódicos Eletrônicos Científicos (PPEC) $)^{11}$, de acesso livre, surgiu da necessidade de se ter, em uma única plataforma, todos os periódicos editados e produzidos no âmbito da universidade.

Quanto à produção compartilhada de artigos, observou-se que não há uma política oficial, pois, na Unesp, cada área tem seu modo de publicação. Na USP, os editores têm autonomia para escolher o que julgam mais adequado entre as diversas formas de produção. Já a Unicamp destacou que valoriza a produção científica resultante de colaborações em diferentes âmbitos, incluindo colaborações acadêmicas, colaborações com o setor empresarial e com o terceiro setor, além daquelas com órgãos governamentais. Uma das marcas apontadas pela Unicamp foi a inovação que, segundo o SIC-Unicamp, tem sido refletida claramente no número de empresas "fillhas" e em suas contribuições com a sociedade. Para o SIC-Unicamp, este é o resultado do estímulo à produção científica por meio de colaborações.

Sobre Dados Abertos, para Santos (2017), existem repositórios e plataformas de dados abertos construídos por centros de dados, financiados e geridos por governos, agências de fomento, editores científicos e instituições acadêmicas e de pesquisa, além de repositórios de áreas especializadas do conhecimento.

Em geral, os repositórios e as plataformas de dados abertos são patrocinados por sociedades ou associações científicas, no intuito de promover infraestruturas para depósito, acesso e compartilhamento de dados governamentais e científicos.

$\mathrm{Na}$ Unesp, o Repositório Institucional é o mesmo ambiente que armazena os Planos de Gestão de Dados e os Dados de Pesquisa. Na Unicamp, políticas referentes a dados de pesquisa são parte do projeto institucional "Gestão de dados de Pesquisa", atualmente em execução e com atividades coordenadas pela Fapesp. Esse projeto tem como um de seus componentes a política institucional associada a dados de pesquisa e se trata de uma tarefa em andamento e de alta complexidade. Em particular, faz-se notar que a Unicamp está implementando, com um grupo de 
universidades parceiras e com o apoio da Fapesp, um repositório de metadados com o objetivo de indexar dados descritores de dados de pesquisa. É importante mencionar que a responsabilidade pelos dados disponibilizados é da universidade que os publicizou. Em 16 de dezembro de 2019, aconteceu o lançamento da primeira versão da Rede de Repositórios de Dados Científicos ${ }^{12}$ associados às pesquisas desenvolvidas em todas as áreas do conhecimento no Estado de São Paulo. A iniciativa envolveu a Unicamp, USP, Unesp, Instituto Tecnológico de Aeronáutica (ITA), Universidade Federal de São Carlos (UFSCar), Universidade Federal do ABC (UFABC), Universidade Federal de São Paulo (Unifesp) e Embrapa Informática Agropecuária (CNPTIA/Embrapa).

De acordo com o site da Coordenação de Aperfeiçoamento de Pessoal de Nível Superior (Capes) (2020, documento eletrônico), Recursos Educacionais Abertos (REA) “[...] são materiais de ensino, aprendizado e pesquisa, em qualquer suporte ou mídia, que estão sob domínio público, ou estão licenciados de maneira aberta, permitindo que sejam utilizados ou adaptados por terceiros.”. A Unesp possui acervo digital com objetos educacionais. Na USP, existem várias iniciativas como REA, que disponibiliza objetos de aprendizagem e Recursos Educacionais Abertos para o ensino. Na Unicamp, o Grupo Gestor de Tecnologias Educacionais (GGTE) tem atuado como parceiro na produção de recursos educacionais abertos, fornecendo estrutura para docentes produzirem recursos digitais em formato audiovisual, além de disponibilizar serviço de streaming para gravação de palestras ou mesmo uma aula. Os conteúdos são posteriormente disponibilizados no YouTube e em uma plataforma de Curso On-line Aberto e Massivo, do inglês Massive Open Online Course (MOOC). Nessa instituição, são utilizados softwares abertos e o Ambiente Virtual de Aprendizagem (AVA) institucional para a elaboração dos recursos educacionais abertos.

A Escola Pragmática preocupa-se em como as novas tecnologias da informação e comunicação são utilizadas para deixar a pesquisa e a produção do conhecimento mais eficientes (CARUSO, 2015), apropriando-se de recursos como blogs, wikis e o potencial das redes sociais, para que a criação do conhecimento possa ser mais eficiente e mais orientada. Com relação ao uso de recursos para inovação aberta, na Unicamp, pode ser destacado o Hackathon em 
Inteligência Artificial Aplicada ao Marketing Business to Business (B2B) que é uma maratona de programação que contou com alunos da graduação e da pósgraduação da própria Unicamp e da Faculdade de Campinas (Facamp). A proposta do desafio foi instigar ideias inovadoras e multidisciplinares que solucionassem ou que promovessem a melhoria dos processos e dificuldades de empresas. Outro projeto adotado na Unicamp foi a maratona para o desenvolvimento de novas soluções em blockchain ${ }^{13}$, com a participação de estudantes, desenvolvedores e pessoas familiarizadas com essa tecnologia, que ganhou espaço no ramo de tecnologia da informação, por servir como base para o desenvolvimento de criptomoedas como o BitCoin.

A Escola da Infraestrutura concentra-se nas plataformas e ferramentas de colaboração, essenciais para a Ciência Aberta. Assim, observa-se o uso do OJS, que é um software de código aberto, criado pelo Public Knowledge Project, para o gerenciamento de periódicos acadêmicos revisados por pares. Entretanto, diversas outras ferramentas podem estar em uso nessas instituições e não foram alcançadas pelo questionário enviado pelo SIC. Ainda, cabe mencionar que não foram alcançados pelas requisições no SIC meios para identificação de ações relacionadas à questão de pessoal, capacitações e outras variáveis que podem ser evidenciadas nesta Escola. É importante relembrar que as Escolas estão interconectadas, podendo as ações de uma refletir nas ações de outra.

Com relação à Escola das Métricas, é importante considerar que o investimento de fundos públicos em pesquisa exige a capacidade de demonstrar claramente retornos benéficos, responsabilidade e bom gerenciamento. A questão de como avaliar a qualidade dos resultados da pesquisa é muito complexa. Existem as métricas formais baseadas em citações que avaliam as atividades no âmbito da academia e métricas que, devido ao apoio das tecnologias e das redes sociais, têm ganhado força e apoio de alguns pesquisadores, as denominadas métricas alternativas, pois se acredita que é essencial o desenvolvimento de indicadores voltados para o impacto social da universidade. De acordo com Mazzitelli (2019, documento eletrônico), ao descrever as palavras de $\operatorname{Prof}^{\mathrm{a}}$. Dr ${ }^{\mathrm{a}}$ Cleópatra da Silva Planeta, Pró-reitora de Extensão Universitária da Unesp, em Workshop sobre indicadores de desempenho da universidade paulista, “[...] os 
rankings privilegiam a questão acadêmica e trazem muito pouco a questão dos indicadores sociais.”, e tal situação deve ser revista.

Quanto ao uso de altmetria, métricas alternativas, a Unesp está iniciando estudos sobre o assunto na instituição, e uma das iniciativas foi uma capacitação intitulada "Altmetrics: métricas alternativas de produção científica", que teve como objetivo apresentar conceitos básicos sobre altmetria, entendida como uma medida alternativa de avaliação do impacto da produção científica e acadêmica, baseada em atividades, ambientes e ferramentas on-line. A USP controla ou acompanha as métricas formais da produção científica dos pesquisadores da instituição por meio das plataformas Web of Science, SciVal, Biblioteca Virtual da Fapesp, Currículo Lattes, Dedalus, Repositório da Produção USP, entre outras. Constantemente, a equipe da Aguia realiza análises bibliométricas, cienciométricas, webométricas e altmétricas, divulgando-as no Jornal da USP, em posts nas redes sociais da Aguia e no seu site oficial.

Na Unicamp, todos os anos, são extraídas informações da produção científica para o preenchimento do Anuário Estatístico da Universidade. Para tanto, se utilizam as ferramentas SciVal (Scopus) e InCites (Clarivate). A Biblioteca fornece informações da produção científica para avaliação institucional, utilizando as mesmas ferramentas citadas. É importante mencionar que a Biblioteca Universitária oferece serviços de apoio à análise métrica da informação científica para suporte à tomada de decisões das unidades de ensino e pesquisa e de outros órgãos da universidade. O monitoramento não é feito de maneira regular, mas sob demanda em alguns momentos do ano, sendo a divulgação realizada no jornal e nas redes sociais da Universidade. A altmetria não é utilizada nessa instituição.

Para Ziegler (2019, documento eletrônico), as três universidades estaduais paulistas têm se movimentado no intuito de "[...] desenvolver novas métricas de avaliação de desempenho e comparações internacionais.", visando à criação de um sistema de uso comum que poderá avaliar não só o desempenho, mas também os impactos socioeconômico, cultural e ambiental das universidades públicas.

Entende-se que o movimento da Ciência Aberta transcende o compartilhamento e o acesso a publicações e aos dados de pesquisas financiadas 
com recurso público. Por meio da abertura do processo científico no todo e da transferência do conhecimento, multiplicam-se os impactos sociais e econômicos da ciência, o que reforça a responsabilidade social científica das instituições (SANTOS, 2017).

\section{Considerações finais}

As universidades investigadas neste estudo apresentam grande foco na pesquisa e, portanto, estão seriamente envolvidas em diversos movimentos voltados para a Ciência Aberta. Ao refletir sobre as ações das universidades estaduais paulistas (USP, Unesp e Unicamp) quanto à divulgação da pesquisa científica e à produção de novos conhecimentos por meio de estratégias voltadas para a abertura da ciência, foi identificado o esforço para dar à sociedade um retorno dos investimentos em pesquisa. Nesse sentido, entende-se que nem todas as iniciativas podem ter sido alcançadas, porém, verifica-se que foi apresentado um rol de ações em direção ao objetivo proposto por este estudo.

Quanto às políticas e iniciativas das universidades estaduais paulistas centradas no acesso aberto, empenhadas em tornar a ciência acessível ao cidadão, pode-se constatar a existência de Repositórios Institucionais consolidados e diversos periódicos em acesso aberto, gerenciados por meio do OJS. Identificouse, ainda, a existência de Portais de Periódicos, embora, com características distintas, pois USP e Unicamp optaram por reunir os títulos em portal único, enquanto na Unesp, cada revista ou conjunto de revistas tem sua própria estrutura de gerenciamento individual. Com relação aos repositórios de dados de pesquisa e Planos de Gestão de dados de pesquisa, há iniciativas para o desenvolvimento de projetos e políticas coordenadas pela Fapesp, demonstrando o importante papel das agências de fomento à pesquisa para o movimento de abertura da ciência. Além disso, observou-se que existem ações quanto à disponibilização de recursos educacionais abertos à comunidade.

Com este estudo, evidenciou-se que as universidades estaduais paulistas, estão definindo algumas ações e diretrizes voltadas para a Ciência Aberta. 
Projetos e políticas estão sendo criados e desenvolvidos em parceria, como é o caso da Política de Dados de Pesquisa das Universidades do Estado de São Paulo.

Destaca-se que projetos de Ciência Cidadã e Inovação Aberta são mais pontuais e dependentes de iniciativas de pesquisadores, não se caracterizando como uma política das instituições, embora tenham sido citadas pesquisas e ações de sucesso das universidades, o que demonstra sensibilidade com essas pautas.

Quanto às métricas de avaliação das publicações, observa-se que ainda estão voltadas para as de natureza formal e que outros formatos estão em discussão. O Scival, a Web of Science e o InCites foram citados como formas de acompanhar a produção científica das publicações institucionais. Já as métricas alternativas, com dados extraídos de redes sociais, ainda são incipientes e pouco utilizadas.

Com relação ao questionário semiestruturado, enviado aos representantes superiores, reitores ou interinos como instrumento de coleta de dados da pesquisa, pondera-se: apesar de ter atendido ao seu propósito neste estudo, reconhece-se que cabem adaptações para o levantamento de mais informações que podem ampliar o diagnóstico e o debate sobre as ações e estratégias adotadas em universidades ou noutras instituições de pesquisa.

Assim, com relação às Escolas Pública e de Infraestrutura, poderão ser identificados: o uso de software livre para gestão dos repositórios; se a administração se compromete com a infraestrutura tecnológica e de pessoal; e se foram previstas as questões de preservação digital das informações e outras que possam surgir na prática da Ciência Aberta.

Uma situação que chamou a atenção foi o fato de duas das instituições terem encaminhado a requisição para que os sistemas de bibliotecas respondessem, apesar de terem sido endereçados aos reitores, responsáveis superiores. Por um lado, ressalta-se que se tratava de questões institucionais e os sistemas não teriam como responder todas as questões apresentadas. Por outro, identifica-se a valorização dos profissionais da área de Biblioteconomia e Ciência da Informação para a abertura da ciência. Destaca-se, assim, um nicho especializado, o qual se entende que deve e pode ser ocupado por esses 
profissionais.

\section{Referências}

ALBAGLI, S. Ciência Aberta em questão. In: SEMINÁRIO

INTERNACIONAL CIÊNCIA ABERTA, QUESTÕES ABERTAS, 1. Rio de Janeiro, 2014. Anais [...]. Rio de Janeiro: Liinc; IBICT; OKF; Unirio, 2014. Disponível em: https://www.cienciaaberta.net/wpcontent/uploads/2014/05/20140820_Albagli_Ciencia_Aberta_em_questao.pdf. Acesso em: 30 jan. 2020.

ALBAGLI, S.; CLÍNIO, A.; RAYCHTOCK, S. Ciência aberta: correntes interpretativas e tipos de ação. Liinc em Revista, Rio de Janeiro, v. 10, n. 2, p. 434-450, 2014. Disponível em: http://revista.ibict.br/liinc/article/view/3593. Acesso em: 30 jan. 2020.

\section{BJÖRNEBORN, L. Small-world link structures across an academic web} space: a library and information science approach. 2004. Tese (Doutorado em Ciência da Informação) - Escola Real de Biblioteconomia e Ciência da Informação, Universidade de Copenhague, Copenhague, 2004. Disponível em: https://komm.ku.dk/ansatte/?pure=files\%2F47039808\%2Flennart_bjorneborn_p hd.pdf. Acesso em: 13 fev. 2020.

BRASIL. Controladoria Geral da União. Guia de procedimentos para atendimento à Lei de Acesso à Informação e utilização do e-SIC. 2. versão. Brasília, 2017. Disponível em: http://www.acessoainformacao.gov.br/lai-parasic/guias-e-orientacoes/guia-de-procedimentos-para-atendimento-a-lei-deacesso-a-informacao-e-utilizacao-do-e-sic. Acesso em: 10 jan. 2020.

BRASIL. Lei $\mathrm{n}^{\circ}$ 12.527, de 18 de novembro de 2011. Regula o acesso a informações previsto no inciso XXXIII do art. $5^{\circ}$, no inciso II do $\S 3^{\circ}$ do art. 37 e no $\S 2^{\circ}$ do art. 216 da Constituição Federal; altera a Lei ${ }^{\circ} 8.112$, de 11 de dezembro de 1990; revoga a Lei $\mathrm{n}^{\circ} 11.111$, de 5 de maio de 2005, e dispositivos da Lei n ${ }^{\circ} 8.159$, de 8 de janeiro de 1991; e dá outras providências. Diário Oficial [da] República Federativa do Brasil, Poder Executivo, Brasília, 18 nov. 2011 - Edição extra. Disponível em:

http://www.planalto.gov.br/ccivil_03/_ato2011-2014/2011/lei/112527.htm. Acesso em: 30 jan. 2020.

BROWN, E.; WOOLSTON, C. Why science blogging still matters: Blogs continue to be an effective platform for communicating your science to major stakeholders - and the public. Nature, [s.l.], v. 554, p. 135-137, 2018. Disponível em: https://www.nature.com/articles/d41586-018-014146 ?utm_source $=$ twitter\&utm_medium $=$ social\&utm_campaign $=$ crs\&utm_content $=100819 v 1$. Acesso em: 13 fev. 2020. 
CARUSO, F. S. Ciência Aberta: ações de pesquisadores acadêmicos na web aberta. 2015. Dissertação (Mestrado em Ciência da Informação) - Programa de Pós-Graduação em Ciência da Informação, Universidade Federal Fluminense, Niterói, 2015.

COORDENAÇÃO DE APERFEIÇOAMENTO DE PESSOAL DE NÍVEL SUPERIOR. Recursos Educacionais Abertos. Brasília, 2020. Disponível em: https://www.capes.gov.br/uab/rea. Acesso em: 2 fev. 2020.

COSTA, M. P.; LEITE, F. C. L. Repositórios institucionais da América Latina e o acesso aberto à informação científica. Brasília: IBICT, 2017. Disponível em_https://repositorio.unb.br/handle/10482/23202. Acesso em: 12 fev. 2020.

CRESWELL, J. W. Investigação qualitativa e projeto de pesquisa escolhendo entre cinco abordagens. 3. ed. Porto Alegre: Penso, 2014.

DENG, F. What is “open"?: An economic analysis of open institutions. In: MPRA Paper $n^{\circ}$ 888. Germany: University Library of Munich, 2008.

ESTELLÉS-AROLAS, E.; GONZÁLEZ-LADRÓN-DE-GUEVARA, F. Towards an integrated crowdsourcing definition. Journal of Information science, [S.1.], v. 38, n. 2, p. 189-200, 2012.

FECHER, B.; FRIESIKE, S. Open Science: one term, five schools of thought. In: BARTLING, S.; FRIESIKE, S. (orgs.). Opening Science: the evolving guide on how the internet is changing research, collaboration and scholarly publishing. New York: Springer, 2014.

FECHER, B.; FRIESIKE, S. Open Science: one term, five schools of thought. The RatSWD Working Papers, [s.1.], n. 218, 2013. Disponível em: https://ssrn.com/abstract=2272036. Acesso em: 13 fev. 2020.

FORESTI, N. A. B. Contribuição das revistas brasileiras de biblioteconomia e ciência da informação enquanto fonte de referência para a pesquisa. Ciência da Informação, Brasília, v. 19, n. 1, 1991. Disponível em: http://revista.ibict.br/ciinf/article/view/375. Acesso em: 13 fev. 2020.

FRANCK, G. et al. Manual de formação em Ciência Aberta. Hanover: Foster, 2018. Disponível em: https://foster.gitbook.io/manual-de-formacao-emciencia-aberta/. Acesso em: 13 fev. 2020.

GOMES, P. C. T. O que é um dashboard?: o guia completo e definitivo! OpServices, Porto Alegre, 16 out. 2017. Disponível em: https://www.opservices.com.br/o-que-e-um-dashboard/. Acesso em: 7 fev. 2020.

GOUVEIA, F. C. Altmetria: métricas de produção científica para além das citações. Liinc em Revista, Rio de Janeiro, v. 9, n. 1, p. 214-227, 2013. 
Disponível em: http://revista.ibict.br/liinc/article/view/3434/3004. Acesso em: 13 fev. 2020.

HADDAWAY, N. R. Open Synthesis: on the need for evidence synthesis to embrace Open Science. Environmental Evidence, [s.l.], v. 7, n. 1, 2018.

HECKER, S. et al. Citizen science: innovation in open science, society and policy. London: UCL, 2018.

JORGE, M. Pesquisa veste aves com mochilas de GPS para entender migração: tecnologia e ciência cidadã ajudam a avaliar impacto da urbanização na rota das aves. Portal de Notícias da Unesp, [s.l.], 2019. Disponível em:

https://www2.unesp.br/portal\#!/noticia/34698/pesquisa-veste-aves-commochilas-de-gps-para-entender-migracao. Acesso em: 2 fev. 2020.

KADIKAR, V. The Wisdom of the crowd: how to democratize derivatives. Blocktelegraph, [s.l.],2019. Disponível em: https://blocktelegraph.io/thewisdom-of-the-crowd-how-to-democratize-derivatives/. Acesso em: 20 jan. 2020.

KNOTH, P.; HERRMANNOVA, D. Towards semantometrics: a new semantic similarity based measure for assessing a research publication's contribution. DLib Magazine, [s.l.], v. 20, n. 11-12, 2014. Disponível em: http://www.dlib.org/dlib/november14/knoth/11knoth.html. Acesso em: 2 fev. 2020.

LAÉ, R. Crowdsoursing: o conceito de inteligência coletiva. 17 maio 2016. Cidade do México. LinkedIn. Disponível em: $<$ https://www.linkedin.com/pulse/crowdsoursing-o-conceito-deintelig\%C3\%AAncia-coletiva-raquel-la\%C3\%A9/?originalSubdomain=pt $>$. Acesso em: 13 fev. 2020.

MAZZITELLI, F. Projeto sobre métricas exercita visão de futuro de universidades. 18 mar. 2019. [s.l.]. Portal de Notícias da Unesp. Disponível em: https://www2.unesp.br/portal\#!/noticia/34399/projeto-sobre-metricasexercita-visao-de-futuro-de-universidades. Acesso em: 13 fev. 2020.

MARQUES, F. Os impactos do investimento. Pesquisa FAPESP, São Paulo, n. 246, 2016. Disponível em: https://revistapesquisa.fapesp.br/os-impactos-doinvestimento/. Acesso em: 30 jun. 2020.

NASSI-CALÒ, L. Avaliação por pares: modalidades, prós e contras. SciELO em Perspectiva, São Paulo, 27 mar. 2015. Disponível em: https://blog.scielo.org/blog/2015/03/27/avaliacao-por-pares-modalidades-pros-econtras/. Acesso em: 2 fev. 2020.

OLIVEIRA, A. C. S.; SILVA, E. M. Ciência Aberta: dimensões para um novo fazer científico. Informação \& Informação, Londrina, v. 21, n. 2, 2016. 
Disponível em:

http://www.uel.br/revistas/uel/index.php/informacao/article/view/27666. Acesso em: 20 jan. 2020.

OLIVEIRA, A. C. S.; GUIMARÃES, P. B. V.; KOSHIYAMA, D. C. A. G. A Ciência Aberta e os direitos de propriedade intelectual: um olhar a partir da economia criativa e da ciência do commons. Revista de Direito da Cidade, Rio de Janeiro, v. 11, n. 1, 2019. Disponível em: https://www.e-

publicacoes.uerj.br/index.php/rdc/article/view/32031. Acesso em: 13 fev. 2020.

OLIVEIRA, D. C. Análise de conteúdo temático-categorial: uma proposta de sistematização. Revista de Enfermagem, Rio de Janeiro, v. 16, n. 4, 2008. Disponível em: http://www.revenf.bvs.br/pdf/reuerj/v16n4/v16n4a19.pdf. Acesso em: 3 fev. 2020.

PASSOS, E. Power BI: Saiba o que é e quanto custa para sua empresa. Infobusines, [s.l.], $1^{\circ}$ jul. 2016. Disponível em: https://infob.com.br/o-que-epower-bi/. Acesso em: 7 fev. 2020.

PAULA, J. A. A extensão universitária: história, conceito e propostas. Interface, Belo Horizonte, v. 1, n. 1, 2013. Disponível em: https://www.ufmg.br/proex/revistainterfaces/index.php/IREXT/article/view/5. Acesso em: 3 fev. 2020.

PRIEM, J. et al. Altmetrics: a manifesto. [s.l.], 26 out. 2010. Disponível em: http://altmetrics.org/manifesto/. Acesso em: 3 fev. 2020.

PRITCHARD, A. Statistical bibliography or bibliometrics? Journal of Documentation, [s.l.], v. 24, n. 4, 1969. Disponível em: https://www.researchgate.net/publication/236031787_Statistical_Bibliography_ or_Bibliometric. Acesso em: 13 fev. 2020.

RUIZ-MALLÉN, I. et al. Citizen science, engagement and transformative learning: a study of the co-construction of a neuroscience research project in Catalonia. Science Communication, [s.l.], v. 38, n. 4, 2016. Disponível em: https://journals-sagepubcom.ez27.periodicos.capes.gov.br/doi/full/10.1177/1075547016642241. Acesso em: 13 ago. 2019.

SANTOS, P. X. (Coord.). Livro verde Ciência Aberta e dados abertos: mapeamento e análise de políticas, infraestruturas e estratégias em perspectiva nacional e internacional. Rio de Janeiro: Fiocruz, 2017.

SANTOS, P. X. A experiência da Fiocruz em gestão da informação. In: SEMINÁRIO TENDÊNCIAS DA GESTÃO DA INFORMAÇÃO EM INSTITUIÇÕES DE C\&T. 1. Brasília, 2013. Anais [...]. Brasília: Embrapa, 2013. Disponível em: https://slideplayer.com.br/slide/1253997/. Acesso em: 13 fev. 2020. 
SÃO PAULO. Decreto do Estado de São Paulo n. 58.052, de 16 de maio de 2012. Regulamenta a Lei federal n. 12.527, de 18 de novembro de 2011, que regula o acesso a informações, e dá providências correlatas. Diário Oficial Poder Executivo - Seção 1, São Paulo, 16 maio 2012. Disponível em: https://www.al.sp.gov.br/repositorio/legislacao/decreto/2012/decreto-5805216.05.2012.html. Acesso em: 31 jan. 2020.

SIGGENER KREIS. Guidelines for good science PR. Berlim: Siggen Circle, 2013. Disponível em: https://www.leopoldina.org/fileadmin/redaktion/Presse/Materialien/Guidelines_ Good_Science_PR.pdf. Acesso em: 13 fev. 2020.

SILVA, F. C. C.; SILVEIRA, L. O ecossistema da Ciência Aberta. Transinformação, Campinas, v. 31, 2019.

SOUZA, I. V. P. Altmetria ou métricas alternativas: conceitos e principais características. AtoZ: novas práticas em informação e conhecimento, [s.l.], v. 4, n. 2, 2015. Disponível em:

https://revistas.ufpr.br/atoz/article/view/44554/27146. Acesso em: 11 fev. 2020.

SUROWIECKI, J. The wisdom of crowds. New York: Anchor Book, 2004. Disponível em: http://www.asecib.ase.ro/mps/TheWisdomOfCrowdsJamesSurowiecki.pdf. Acesso em: 12 fev. 2020.

UNDERWOOD, S. Blockchain beyond bitcoin. Communications of the ACM, New York, v. 59, n. 11, 2016, p. 15-17. Disponível em: https://dl.acm.org/doi/fullHtml/10.1145/2994581. Acesso em: 11 abr. 2020.

UNIVERSIDADE DO MINHO. Projeto Open Science. Minho, 2020. Disponível em: https://openaccess.sdum.uminho.pt/?page_id=276. Acesso em: 12 fev. 2020.

UNIVERSIDADE ESTADUAL PAULISTA "JÚLIO DE MESQUITA FILHO". Portaria Unesp-257, de 25 de julho de 2019. Regulamenta, no âmbito da Universidade Estadual Paulista "Júlio de Mesquita Filho" - Unesp, a Lei 12.527, de 18-11-2011, que regula o acesso a informações. Diário Oficial - Poder Executivo - Seção 1, São Paulo, v. 129, n. 139, 26 jul. 2019. p. 53. Disponível em: https://ape.unesp.br/pdf_siteape/PortariaUNESP257-19.pdf. Acesso em: 31 jan. 2020.

ZIEGLER, M. F. Universidades fixam novas métricas de desempenho acadêmico. Agência Fapesp, São Paulo, 2019. Disponível em: https://www2.unesp.br/portal\#!/noticia/35179/universidades-fixam-novasmetricas-de-desempenho-academico. Acesso em: 2 fev. 2020.

ZIVKOVIC, B. Science Blogs: definition, and a history. Scientific American, [s.l.], 10 jul. 2012. Disponível em: https://blogs.scientificamerican.com/a-blog- 
around-the-clock/science-blogs-definition-and-a-history/?print=true. Acesso em: 12 fev. 2020.

\title{
Actions and strategies focused on the open science ecosystem in são paulo state universities: a multicase-study
}

\begin{abstract}
This research aims to identify and reflect on the actions of the state universities in São Paulo - University of São Paulo (USP), State University "Júlio de Mesquita Filho" (Unesp) and State University of Campinas (Unicamp) - regarding the dissemination of scientific research and the production of new knowledge through strategies focused on Open Science. As specific objectives, it is intended to point out public policies adopted in the state universities of São Paulo and to identify how these institutions define their guidelines related to new forms of data and information sharing. As data collection procedures, bibliographic research and documentary survey are used. In addition, we sent to the managers of the three selected universities, via the Citizen Information Service (SIC), a request to share information about the Open Science initiatives and strategies developed by these institutions. For the analysis, we categorized and classified the data collected through the five currents of thought that represent perspectives recognized by the authors Fecher and Friesike (2013). The data were treated in an Excel spreadsheet and interpreted with the help of a set of tools in the Power BI business intelligence clouds. It was concluded that the investigated universities have a great focus on research and, therefore, are seriously involved in several movements focused on Open Science.
\end{abstract}

Keywords: Open Science. São Paulo State Universities - Brazil. Scholarly Communication.

Recebido: $16 / 04 / 2020$

Aceito: $24 / 07 / 2020$

\section{Declaração de autoria \\ Concepção e elaboração do estudo: \\ Coleta de dados: \\ Análise e discussão de dados: \\ Redação e revisão do manuscrito:}

\section{Como citar}

RIBEIRO, Nivaldo Calixto; OLIVEIRA, Dalgiza Andrade; SANTOS, Sarah Rúbia de Oliveira. Ações e estratégias voltadas para a ciência aberta em universidades estaduais paulistas: um estudo multicaso. Em Questão, Porto Alegre, v. 27, n. 2, p. 164-192, abr./jun. 2021.

Doi: http://dx.doi.org/10.19132/1808-5245272.164-192

\footnotetext{
1 As universidades aparecem no Times Higher Education World University Rankings 2020, no CWTS Leiden Ranking 2019, no QS World University Ranking 2019, no Academic Ranking of World Universities 2019 (ARWU) e no Ranking Universitário da Folha.

2 Crowdsourcing é definido por Estellés-Arolas e González-Ladrón-de-Guevara (2012) como a terceirização, pela internet, de tarefas para uma multidão claramente definida que recebe uma recompensa em troca de sua colaboração. Estratégia que também alinha-se à tese defendida por Surowiecki (2004), de
} 
que nas circunstâncias certas, a multidão eclética toma decisões mais inteligentes do que grupos de especialistas.

3 UNIVERSIDADE ESTADUAL PAULISTA "JÚLIO DE MESQUITA FILHO". Serviço de Informações ao Cidadão. São Paulo, 2020. Disponível em: https://ape.unesp.br/sic/. Acesso em: 30 jan. 2020.

${ }^{4}$ UNIVERSIDADE DE SÃO PAULO. Serviço de Informações ao Cidadão. São Paulo, 2020. Disponível em: https://uspdigital.usp.br/sic/faleConosco?codmnu=6658. Acesso em: 31 jan. 2020.

${ }^{5}$ UNIVERSIDADE ESTADUAL DE CAMPINAS. Serviço de Informações ao Cidadão. Campinas, 2020. Disponível em: https://www.unicamp.br/unicamp/informacao/formulario-para-pedido-de-acessoinformacao-pf. Acesso em: 31 jan. 2020.

${ }^{6}$ Aves da cidade, espécies migratórias que usam parques urbanos na Mata Atlântica. Site disponível em: https://avesdacidade.wordpress.com/quem-sou/publicacoes/. Acesso em: 2 fev. 2020.

7 Repositório Institucional da UNESP. Disponível em: https://repositorio.unesp.br/.

${ }^{8}$ Repositório Institucional da USP. Disponível em: https://bdpi.usp.br/.

${ }^{9}$ Repositório Institucional da UNICAMP. Disponível em: http://repositorio.unicamp.br/.

${ }^{10}$ O Portal de Revistas da USP está disponível em: http://www.revistas.usp.br/wp/. Acesso em: 2 fev. 2020.

11 O Portal de Periódicos Eletrônicos Científicos (PPEC) da Unicamp, está disponível em: https://periodicos.sbu.unicamp.br/ppec/. Acesso em: 2 fev. 2020.

12 Disponível em: https://metabuscador.uspdigital.usp.br/. Acesso em: 28 fev. 2020.

13 “O Blockchain, como a Internet, é uma infraestrutura global aberta que permite que empresas e indivíduos que fazem transações cortem o intermediário, reduzindo o custo das transações e o lapso de tempo de trabalho com terceiros" (UNDERWOOD, 2016, p. 15). 